\title{
Correlational study on Hypertension and Dietary Regimen among young adults in Bontoc, Mountain Province Philippines
}

\author{
June II A. Kiblasan *1, Lucia T. Payagen ${ }^{2}$, Rebecca E. Dulnuan², Evangeline C. Singson ${ }^{3}$, Potenciano P. Uy ${ }^{1}$ \\ ${ }^{1}$ Faculty of Nursing, Misurata University, Misurata, Libya \\ ${ }^{2}$ Faculty of Nursing, University of Tripoli, Tripoli, Libya \\ ${ }^{3}$ Faculty of Languages, University of Tripoli, Tripoli, Libya
}

Received: April 12, 2015

Accepted: May 18, 2015

Online Published: June 2, 2015

DOI: $10.5430 /$ cns.v3n4p1

URL: http://dx.doi.org/10.5430/cns.v3n4p1

\begin{abstract}
Interest in the relationship between hypertension and dietary regimen is an issue that needs to be understood for it has recently intensified that hypertension and non-optimal blood pressure levels are the most important contributors to cardio and cerebrovascular disease that is common not only to Filipinos but in the Pacific region with prevalence that is analogous to what is reported in developed countries. Thus, this needs an attention in which lifestyle would benefits these people. As such, diet is one of the important but neglected as regimen. With this, the authors agreed to come up with a study to look for the level of awareness of fifty hypertensive young adults on hypertension and their compliance to dietary regimen and at the same time, to uncover if there is significant relationships between the level of awareness on hypertension and the level of compliance to dietary regimen. Descriptive correlational study was employed and a reliable questionnaire that was formulated and utilized as the data gathering tool. Hypertensive young adults were found to be much aware on hypertension and they are high compliant to dietary regimen. There is significant relationship between the level of awareness on hypertension and level of compliance to dietary regimen.
\end{abstract}

Key Words: Hypertension, Hypertensive young adults, Awareness, Compliance, Dietary regimen

\section{INTRODUCTION}

The world is moving in a fast pace. There are targets to be achieved and works to be done. Too much is to be accomplished in too little time. There is stiff competition and survival of the fittest rule. These give way to the incessant response of the people, physiologically and psychologically. Anxiety, stress, unhealthy diet and lifestyles are becoming part and parcel of our daily routine. All this put a lot of effect on today's classes and masses. And on personal level, it results into hypertension. Hypertension is defined as a systolic blood pressure (SBP) equal or more than $140 \mathrm{mmHg}$ and/or diastolic blood pressure (DBP) equal or more than $90 \mathrm{mmHg}$ based on the mean of 2 or more properly measured seated blood pressure readings on each of 2 or more office visits by the seventh report of the Joint National Committee on Prevention, Detection, Evaluation, and Treatment of High Blood Pressure (JNC) ${ }^{[1]}$ Hypertension can be defined as blood pressure (BP) consistently $\geq 140 / 90 \mathrm{mmHg}$, it is considered to be one of the most prevalent and serious risk factors for cardiovascular disease. ${ }^{[2]}$ Hypertension is a major risk factor for stroke and coronary heart disease. ${ }^{[3,4]}$ A large percentage of the population, however, are unaware that they

*Correspondence: June II A. Kiblasan; Email: jiiak0321@yahoo.com; Address: Faculty of Nursing, Misurata University, Misurata, Libya. 
have hypertension, and those known to be hypertensive are often not adequately controlled. ${ }^{[5-7]}$ The first line of treatment for hypertension is preventive lifestyle changes and medication, ${ }^{[8,9]}$ and poor control is attributed to poor compliance with the treatment regime. ${ }^{[10-12]}$ Sackett and Haynes ${ }^{[13]}$ defined patient compliance as "the extent to which a person's behavior in terms of taking medication, following diet, or executing life-style changes coincides with medical or health advice". Compliance can be viewed as a patient's behavior in terms of timeliness in seeking care, attendance at follow-up appointments or observance of the physician's advice. As compliance improves the outcome of hypertension, understanding its pattern is an important step in evaluating the effect of a hypertension treatment regimen.

A study done in 2005 on Global burden of hypertension, indicates that more than a quarter of the world's adult total population is nearly one billion had hypertension in 2000, and that this proportion will increase to $29 \%$ ( 1.56 billion) by $2025 .^{[14]}$ On the other way around, a healthy diet is beneficial in the treatment of hypertension in which having a low-salt diet can decrease systolic blood pressure by 2-8 $\mathrm{mmHg}$. Dietary Approaches to Stop Hypertension (DASH) emphasize that using fruits and vegetables, low-fat diets, and reduced saturated fats can decrease systolic blood pressure by about $8-14 \mathrm{mmHg} .{ }^{[15]}$ Dietary treatment is recommended for a wide diabetes and hypertension, a low-cholesterol diet can help prevent future complications, in some others like celiac disease, and diet has immediate effects. Dietary interventions are usually considered as the first therapeutic choice because of their lower economic burden and side effects compared to drug interventions. However, patients do not usually adhere to dietary interventions without immediate effects. Therefore, patients and health professionals prefer to take secondary treatments such as medications or even surgery. ${ }^{[16]}$ However, it suggests that men and women will have similar overall prevalence of hypertension and that such prevalence increase with age consistently in all world regions. People from all age groups can suffer from this health problem. In spite of that, many people do not know that they suffer from hypertension for it silently affects our vital organs. With this, it is a mandate to promote and protect healthy lifestyles. For common understanding, healthy lifestyle has been operationally defined as a way of life that promotes and protects health and well-being. This would include practices that promote health such as healthy diet and nutrition. Dietary modification is often discussed with patients and can provide a great benefit in blood pressure regulation. As such, reviewing the current evidence will be very useful in guiding patients and their physician and/or dietician in decision making. In this view, the authors aimed to come up with a finding if how do hypertensive young adults are aware regarding hypertension and their compliance to dietary regimen according to gender that can be used in developing a plan of care to control hypertension and at the same time, to examine if there is significant relationship in between.

\section{Methods}

Descriptive correlational design was utilized and purposive sampling was employed. The sources of data were twenty nine females and twenty one males who are both hypertensive young adults according to the Erik Erikson's stages of human development which are from age of 20 to 40 years old that made the total number of fifty respondents. A questionnaire served as the instrument that was given to the respondent has two parts in which the first part includes indicators that will assess level of awareness on hypertension and the second part refers to the indicators on level of compliance to dietary regimen that was subjected to content validation and reliability testing ( $\mathrm{rK}-\mathrm{R}=0.909$ ). Likert Scale was used to quantify the responses of the respondents. Collected data were tallied and subsequently subjected to statistical treatment using the Statistical Package for Social Sciences Version 20.0 (SPSS) employing Pearson product-moment correlation coefficient (PPMCC) to test for the significant relationship in between the level of awareness on hypertension and compliance to dietary regimen.

\section{RESULTS AND Discussion}

Awareness to facts about hypertension is essential not only to hypertensive clients but to the entire populace as it has been reported that billions of individuals are suffering from hypertension. In this study, it was a decision to accommodate all reported total number of young adults with hypertension in three barangays namely Calutit, Bontoc Ili, Samoki, and Poblacion that encompasses the town proper of Bontoc, Mountain Province, Philippines. Home visits and follow ups were conducted to determine the actual number of respondents with the following limitations: Rest for at least five minutes before taking the blood pressure; Systolic Pressure of $\geq 140 \mathrm{mmHg}$ and Diastolic Pressure is $\geq 90 \mathrm{mmHg}$ taken in three consecutive periods; and no family nor medical related history of hypertension. However, not all target population were available because some are in the farm, migrated to nearby places to work with undetermined date of returning home, and cannot be located anymore of unknown reason. There are fifty hypertensive young adults that were accommodated and majority of them belongs to age from 30 to 40 years old that covers the $90 \%$ of the total respondent while the remaining $5 \%$ is from the age of 25 to 29 . The finding shows that as an individual grows older, the possibility of having hypertension is increasing. On the other 
hand, Table 1 shows how young adults in Bontoc, Mountain Province, Philippines are aware on one of the leading causes of heart disease. Hypertensive young adults were found to be much aware on hypertension with over-all weighted means of 4.04 for males and 4.08 for females. Statistically, though hypertensive young adult were much aware on facts about hypertension, still females need to be educated in terms of maintaining a normal blood pressure which is the most important preventive measure for hypertension and the common manifestations such as nape pain, headache, dizziness and chest pain because there are times that it was perceived as work related and hormonal problems. Same as to male young adults, regular medical consultation helps in the early detection of hypertension and common manifestations such as nape pain, headache, dizziness and chest pain were at the least according to its weighted means. They have to be enlightened on the matter for most of the time; they are busy with their work as the breadwinner of the family. Yet, such manifestations were considered as ordinary for them and were considered as work related problem. The result is related but not totally similar to a study ${ }^{[17]}$ that has demonstrated inadequate knowledge of hypertension among hypertensive patients where conscious efforts should be made and time set aside to health educates hypertensive patients. Organization of "hypertensive club or society" could be encouraged. These will reduce dissemination of false or inaccurate information by hypertensive patients to the public and its attendant dangers. Another shared study concluded that it is urgently necessary to promote knowledge, awareness, and health literacy among the ethnic Karen tribes to prevent hypertension and associated cardiovascular diseases (CVDs). ${ }^{[18]}$ On the other hand, a result from ${ }^{[19]}$ suggested that, although general knowledge and awareness of hypertension is adequate, patients do not have a comprehensive understanding of this condition. For instance, patients do not recognize the importance of elevated SBP levels or the current status of their BP control. An opportunity exists to focus patient education programs and interventions on the cardiovascular risk associated with uncontrolled hypertension, particularly elevated SBP levels. This finding is similar to the findings of this endeavor.

Table 1. Awareness on Hypertension

\begin{tabular}{|c|c|c|c|c|}
\hline \multirow{2}{*}{ Facts about Hypertension } & \multicolumn{2}{|c|}{ Female } & \multicolumn{2}{|l|}{ Male } \\
\hline & WM & DE & WM & DE \\
\hline Hypertension is a condition which there is an increase in the blood pressure & 4.07 & MA & 4.14 & MA \\
\hline I can maintain a normal blood pressure through health lifestyles & 3.90 & MA & 4.14 & MA \\
\hline Blood pressure of $140 / 90 \mathrm{mmHg}$ or greater taken daily for three times is considered as hypertensive & 4.03 & MA & 4.19 & MA \\
\hline Genetics, obesity and the type of diet increases the risk of having hypertension & 4.14 & MA & 4.05 & MA \\
\hline Stress, smoking, and alcohol intake are lifestyle factors that affect blood pressure & 4.10 & MA & 4.10 & MA \\
\hline Maintaining a healthy diet and adequate rest and sleep are preventive measure for hypertension & 4.17 & MA & 4.05 & MA \\
\hline Regular exercise can reduce the risk of developing complication of hypertension & 4.00 & MA & 4.10 & MA \\
\hline Stroke, renal insufficiency and coronary heart disease are complications of uncontrolled hypertension & 4.00 & MA & 4.14 & MA \\
\hline Regular medical clinic consultation helps in early detection of hypertension & 4.03 & MA & 3.95 & MA \\
\hline Nape pain, headache, dizziness and chest pain are common manifestations of hypertension & 3.93 & MA & 3.95 & MA \\
\hline Average Weighted Mean (AWM) & 4.04 & MA & 4.08 & MA \\
\hline
\end{tabular}

Note: Fully Aware $(\mathrm{FA})=4.21-5.00$; Much Aware $(\mathrm{MA})=3.41-4.20$; Partially Aware $(\mathrm{PA})=2.61-3.40$; Less Aware $(\mathrm{LA})=1.81-2.60$; Not Aware $(\mathrm{NA})=1.00-1.80$; WM = Weighted Mean; $\mathrm{DE}=$ Descriptive Equivalent

Dietary regimen is a kind of management that is applicable to any disease where diet is one of the concerns. With this study, dietary regimen is essential since substances or biochemical found in foods we are eating affect our circulation that can cause hypertension. Table 2 presents the compliance of hypertensive young adults to dietary regimen of hypertension. Generally, both hypertensive male and female young adults are highly compliant to dietary regimen of being hypertensive with average weighted means of 3.98 and 4.00 respectively. Taking into consideration this kind of health related problem, dietary regimen should not be taken for granted but instead, it should be seriously and religiously complied. It is a common culture of Filipinos to eat high fatty foods in a deep fried or in roasted manner which reflects the reality in the findings most especially women. In mountain province, they have the culture of preparing smoked meat called "etag" that can affect individual's health if eaten frequently. Generally, Filipinos are fond of preparing fatty foods most especially during holidays and special occasions. This is opposite with the finding of a study conducted where Patients with hypertension were poorly compliant with exercise and dietary regimens. ${ }^{[20]}$ On the other hand, a similar 
study $^{[21]}$ though this is on compliance to medication has a little bit similarity in findings where there is good compliance with drug treatment that was observed in $54.2 \%$ of the respondents and poor compliance among the remainder but poor compliance was found due to ignorance on need for regular treatment $(32.7 \%)$, lack of funds to purchase drugs $(32.7 \%)$ and side effects of drugs $(12.1 \%)$. Patients with formal education, and higher monthly income were more compliant to treatment. In addition, those on single drugs were more compliant compared to those on two or more drugs and poor compliance was found to be mainly due to ignorance and lack of funds to purchase drugs. Another similar study ${ }^{[22]}$ but of opposite finding revealed that hypertensive patients are not following dietary regimen and they are they have the same on food control pattern. Lifestyle modification and adherence to DASH diet whereas eating more fruits and vegetables, reducing salt and fat intake, low simple carbohydrate consumption, losing weight, regular exercise, stop smoking, decrease alcohol intake, and managing stress are highly recommended to hypertensive adults that is beneficial in lowering BP. These non-pharmacological measures can be sufficient to control BP or to decrease the amount of required medications, and to prevent, treat or remove associated risk factors, reduce CVD risk, diabetes, and obesity.

Table 2. Compliance to dietary regimen

\begin{tabular}{|c|c|c|c|c|}
\hline \multirow{2}{*}{ Dietary Regimen } & \multicolumn{2}{|c|}{ Female } & \multicolumn{2}{|l|}{ Male } \\
\hline & WM & DE & WM & DE \\
\hline I eat soy beans products tofu and legumes such as string beans and others & 4.00 & HC & 3.95 & HC \\
\hline I eat defatted lean meat and skinless chicken & 3.93 & $\mathrm{HC}$ & 4.05 & $\mathrm{HC}$ \\
\hline I eat non-fat dairy products such as non-fat cheese and milk & 3.90 & $\mathrm{HC}$ & 4.10 & HC \\
\hline I eat grain and grain products such as wheat bread, cereal, pasta and oat meals & 4.03 & $\mathrm{HC}$ & 4.10 & $\mathrm{HC}$ \\
\hline I eat yellow and green leafy vegetables such as carrots, cabbage, "pechay”, squash and "kangkong” & 4.00 & HC & 4.10 & HC \\
\hline I eat fruits such as apple, avocado, melon, orange, papaya and grapes & 4.00 & $\mathrm{HC}$ & 3.95 & HC \\
\hline I don’t eat food high in cholesterol like "lechon”, "kare-kare” and deep fried foods like fish and chicken & 3.97 & $\mathrm{HC}$ & 3.90 & HC \\
\hline I don't eat canned goods such as corned beef, sardines and sausage & 3.97 & HC & 4.05 & HC \\
\hline I don’t eat foods high I salt like “bagoong”, dried fish and smoked fish & 4.97 & $\mathrm{HC}$ & 3.90 & $\mathrm{HC}$ \\
\hline I don't eat foods that contain baking soda like pan cake and waffles & 3.90 & HC & 3.95 & HC \\
\hline Average Weighted Mean (AWM) & 3.98 & HC & 4.00 & HC \\
\hline
\end{tabular}

Note: Very High Compliance $(\mathrm{VHC})=4.21-5.00$; High Compliance $(\mathrm{HC})=3.41-4.20$; Moderate Compliance $(\mathrm{MC})=2.61-3.40$; Less Compliance $($ LC $)=1.81-2.60 ;$ Not Compliance $(\mathrm{NC})$ = 1.00-1.80; WM = Weighted Mean; $\mathrm{DE}=$ Descriptive Equivalent

Table 3. Relationship between awareness on Hypertension and compliance to dietary regimen

\begin{tabular}{llll}
\hline Respondents & Level of Awareness on Hypertension & Level of Compliance on Dietary Regimen & Combined Weighted Mean \\
\hline Male & 4.08 & 4.00 & 4.04 \\
Female & 4.04 & 3.98 & 4.01 \\
Total Weighted Mean & 4.06 & 3.99 & 4.025 \\
\hline
\end{tabular}

Compliance to dietary and awareness on hypertension is an issue that will elicit an idea of any individual of thinking how it would be or do these can influence each other? Are both has connection? A heart-healthy diet is important for managing blood pressure problem that possibly helps in reducing risk of heart attack, heart disease, stroke and other diseases. That is why other people prefer to have a diet plan and for those who are having hypertension, DASH or Dietary approaches to stop hypertension and some preferred health education. But at the end, self-discipline on complying with regimen is the best thing to consider in managing hypertension. Table 3 will prove if there is relationship between awareness on hypertension and compliance to dietary regimen among hypertensive young adults in Bontoc, Mountain Province, Philippines. With the use of computed means of males and females young adults, the Pearson's $r$ is $-1.000 ; n=2 ; p=$ .000 . With these values, the correlation between awareness on hypertension and the compliance to dietary regimen is significant at the 0.01 level (2-tailed). This means that there is a strong relationship between awareness on hypertension and 
compliance to dietary regimen among hypertensive young adults in Bontoc, Mountain Province, Philippines that the higher the level of compliance to dietary regimen, the lower the blood pressure it may do. The findings of having positive relationship is similar to the conducted study that there were significant relationships between patients' drug adherence and different levels of income, number of pills taken each day, and the frequency of blood pressure control. ${ }^{[23]}$ Another study results demonstrate that, compared with the control diet, use of the DASH diet lowered SBP by $11.2 \mathrm{mmHg}$ in participants with stage 1 Isolated Systolic Hypertension (ISH). This was sufficient to control SBP to $<140 \mathrm{mmHg}$ in 18 of 23 participants in the DASH diet group. This treatment effect size is comparable to the blood pressure reduction seen with a typical antihypertensive drug. For example, if we adjust for changes in the control diet group, the DASH diet effect was $-11.2 /-4.5 \mathrm{mmHg}$. ${ }^{[24]}$

\section{Conclusion}

Awareness on hypertension and compliance to dietary regimen is an important concern on preventing occurrence of hypertension and its complication. As per statistical survey that was carried and the findings made to assess awareness and compliance of hypertensive young adults in Bontoc, Mountain Province, Philippines, the following conclusions were made: (1) Majority of the hypertensive young adults are with an age ranges from 30 to 40 years old. (2) Hypertensive young adults are much aware on facts regarding hypertension but attitude and behavior sometimes affects their awareness leading to ignore such signs of being hypertensive. (3) Hypertensive young adults are highly compliant with dietary regimen but there are times that culture, traditions and practices intervene on its sustenance. (4) There is a strong relationship between awareness on hypertension and compliance to dietary regimen among hypertensive young adults.

\section{ACKNOWLEDGEMENTS}

The authors would like to express their warm heartfelt gratitude to the concerned citizens of the place this endeavor was conducted for sharing us the data needed in its accomplishment. We thank our friends and families who provided their moral supports, insights and expertise that greatly assisted and for sharing their pearls of wisdom with us during the course of this research.

Source of Funding: This research has no specific grant from any funding agency in the public, commercial or not-forprofit sectors. It was supported by the authors.

Ethical Clearance: This research was conducted with the approval of concerned citizens in the place where the research was conducted. Confidentiality of the names and other personal information of the respondents were maintained.

\section{CONFLicts OF INTEREST Disclosure}

There is no professional, personal or family allegiance, bias, inclination, obligation or loyalty which may in any way affect the objectivity, independence or impartiality in making this research.

\section{REFERENCES}

[1] Chobanian AV, Bakris GL, Black HR, et al. Seventh report of the joint national committee on prevention, detection, evaluation, and treatment of high blood pressure. Hypertension. 2003; 42(6): 12061252. PMID:14656957. http://dx.doi.org/10.1161/01. HYP. $0000107251.49515 . c 2$

[2] Miller H, Berra K, Long J. Awareness, understanding, and treatment of previously diagnosed hypertension in baby boomers and seniors: a survey conducted by harris interactive on behalf of the preventive cardiovascular Nurses association. 2009; 12(5): 328-334.

[3] Al-Nozha MM, Arafah MR, Al-Marou YY, et al. Coronary artery disease in Saudi Arabia. Saudi Med J. 2004; 25: 1165-1171. PMID: 15448760 .

[4] Qari FA. Profile of stroke in a teaching university hospital in Western Region. Saudi Med J. 2000; 21: 1030-1033. PMID:11360063.

[5] Al-Rukban MO, Al-Sughair AM, Al-Bader BO, et al. Management of hypertensive patients in primary health care setting: auditing the practice. Saudi Med J. 2007; 28: 85-90. PMID:17206296.

[6] Al-Tuwijri AA, Al-Rukban MO. Hypertension control and comorbidities in primary health care centers in Riyadh. Ann Saudi Med. 2006; 26: 266-271. PMID:16883084.

[7] Hajjar I, Kotchen TA. Trends in prevalence, awareness, treatment, and control of hypertension in the United States. JAMA. 2003; 290: 199-206. PMID:12851274. http://dx.doi.org/10.1001/jama. 290.2 .199

[8] Okonofua EC, Simpson KN, Jesri A, et al. Therapeutic inertia is an impediment to achieving the Healthy People 2010 blood pressure control goals. Hypertension. 2006; 47: 345-351. PMID:16432045. http: //dx.doi.org/10.1161/01.HYP.0000200702.76436.4b

[9] Law M, Wald N, Morris J. Lowering blood pressure to prevent myocardial infarction and stroke: a new preventive strategy.Health Technol Assess. 2003; 7(31): 1-94. PMID:14604498. http://dx.doi .org/10.3310/hta7310

[10] World Health Organization. Arterial hypertension, Geneva, 1978 Technical Report Series 628.

[11] Chobanian AV, Bakris GL, Black HR, et al. Seventh report of the Joint National Committee on prevention, detection, evaluation, and treatment of high blood pressure. Hypertension. 2003; 42: 1206-1252. PMID:14656957. http://dx.doi.org/10.1161/01.HYP.0000 107251.49515.c2

[12] Neter JE, Stam BE, Kok FJ, et al. Influence of weight reduction on blood pressure: a meta-analysis of randomized controlled tri- 
als. Hypertension. 2003; 42: 878-884. PMID:12975389. http:

//dx.doi.org/10.1161/01.HYP.0000094221.86888.AE

[13] B Haynes, DW Taylor, DL Sackett. Conceptual and methodologic problems in measuring patient compliance: Compliance in health care. Maryland: The John Hopkins University Press; 1979. 23-45 p.

[14] Kearney P, Whelton M, Reynolds KM, et al. Global burden of hypertension: analysis of worldwide data. Lancet. 2005; 365: 217-23. http://dx.doi.org/10.1016/S0140-6736(05)70151-3

[15] Braunwald E, Zipes DP, Libby P, et al. Braunwald's heart disease: a textbook of cardiovascular medicine. 7th ed. Philadelphia, PA: Elsevier Saunders; 2004.

[16] Bosworth HB, Oddone EZ, Weinberger M. Patient Treatment Adherence: Concepts, Interventions, and Measurement. 1st ed. London, UK: Routledge; 2006.

[17] Familoni B Oluranti, Ogun S Abayomi, Aina A Olutoyin. Knowledge and awareness of hypertension among patients with systemic hypertension, Journal of the National Medical Association. 2004 May; 96(5): 620-624. PMID:15160976. http://www.ncbi.nlm.n ih.gov/pmc/articles/PMC2640651/

[18] Aung MN, Lorga T, Srikrajang J, et al. Assessing awareness and knowledge of hypertension in an at-risk population in the Karen ethnic rural community, Thasongyang, Thailand. International Journal of General Medicine. 2012; 2012(5): 553-561. http://dx.doi .o rg/10.2147/IJGM.S29406
[19] Oliveria SA, Chen RS, McCarthy BD, et al. Hypertension Knowledge, Awareness, and Attitudes in a Hypertensive Population. Journal of General Internal Medicine. 2005 Mar; 20(3): 219-225. http: //dx.doi.org/10.1111/j.1525-1497.2005.30353.x

[20] Manal Ibrahim Hanafi Mahmoud, Clinical Study Compliance with treatment of patients with hypertension in Almadinah Almunawwarah: A community-based study. Journal of Taibah University Medical Sciences. 2012; 7(2): 92-98. http://dx. doi.org/10.10 $16 / j \cdot j$ tumed.2012.11.004

[21] Kabir M, Iliyasu Z, Abubakar IS, et al. Compliance to medication among hypertensive patients in Murtala Mohammed Specialist Hospital, Kano, Nigeria. Journal of Community Medicine \& Primary Health Care. 16(1): 16-20. http://www.ajol.info/index.php/ jcmphc/article/viewFile/32401/6069

[22] Yehia A, Seham AH, Amal J. Nutritional Status and Diet Intake among Hypertensive Palestinians in Gaza Strip, Merit Research Journal of Medicine and Medical Sciences. 2015; 3(2): 27-35. http://www.meritresearchjournals.org/er/index.htm

[23] Zabihi RE, Ashktorab T, Banaderakhshan H, et al. Adherence to therapeutic regimens in patients with hypertension. ARYA Atherosclerosis Journal. 2012: 8(Special Issue in National Hypertension Treatment): S190-S194.

[24] Moore TJ, Conlin PR, Ard J, et al. DASH (Dietary Approaches to Stop Hypertension) Diet Is Effective Treatment for Stage 1 Isolated Systolic Hypertension. American Heart Association. http: //hyper.ahajournals.org/content/38/2/155.full 